\title{
Ampliação da área ocorrência de Drosophila suzukii (Diptera, Drosophilidae) em Santa Catarina, Brasil
}

\author{
Enlargement of occurrence area of Drosophila suzukii (Diptera, Drosophilidae) in Santa Catarina, \\ Brazil
}

\section{Luiza Cristiane Fialho Zazycki ${ }^{1 *}$, Julia Patricia Vargas², Vanessa Santana Bonfim² e Flávio Roberto Mello Garcia ${ }^{3}$}

\author{
1Universidade Estadual de Mato Grosso do Sul, Aquidauana, MS, Brasil. *Autor para correspondência: luizazazycki@gmail.com. \\ ${ }^{2}$ Universidade Federal de Santa Catarina, Curitibanos, SC, Brasil. \\ 3Universidade Federal de Pelotas, Pelotas, RS, Brasil.
}

Submissão: 15/06/2018 / Aceite: 01/07/2019

\begin{abstract}
RESUMO
A drosófila-de-asa-manchada possui elevada importância econômica por danificar frutos de epicarpo delgado os quais possuem elevado valor comercial agregado, principalmente para o consumo in natura. Os relatos da praga mostram que ela está distribuída em diferentes locais estados do Brasil (Rio Grande do Sul, Santa Catarina, Paraná, São Paulo, Rio de Janeiro, Distrito Federal e Minas Gerais). Nosso estudo teve como objetivo ampliar a área de ocorrência da espécie na região Serrana de Santa Catarina. Para o monitoramento de sua ocorrência foram utilizadas armadilhas tipo PET, iscadas com vinagre de maçã em um cultivo orgânico de morango conduzido em sistema semi-hidropônico no município de Frei Rogério. As armadilhas monitoraram 8.600 plantas durante dez dias e o material coletado foi avaliado em microscópio estereoscópico. Foram coletados diversos insetos de Drosophilidae e destes, noventa e oito fêmeas e quatro machos de Drosophila suzukii revelando assim a presença da praga na região Serrana de Santa Catarina.
\end{abstract}

PALAVRAS-CHAVE: drosófila-de-asa-manchada, morango, pequenas frutas.

\section{ABSTRACT}

The spotted wing Drosophila has significant economy relevance due to attacking thin epicarp fruits, which have high commercial value, mainly for "in natura" consumption. The Brazilian pest reports to date are distributed in the states of Rio Grande do Sul, Santa Catarina, Paraná, São Paulo, Rio de Janeiro, Goiás, and Minas Gerais. Our study aimed to record the enlargement of occurrence area of this species in the Serrana region of Santa Catarina. For this, traps PET type baited with apple cider vinegar in an organic strawberry crop in a semi-hydroponic system in the municipality Frei Rogério. Traps monitored 8.600 plants for ten days, and the collected material was evaluated with a stereoscopic microscope. Several insects of Drosophilidae family were collected, of which ninety-eight females and four males of Drosophila suzukii, thus revealing the enlargement of pest presence in the Serrana region of Santa Catarina.

KEYWORDS: spotted wing Drosophila, strawberry, berries.

A drosófila-da-asa-manchada, Drosophila suzukii (Matsumura) (Diptera: Drosophilidae) é uma espécie invasora, proveniente do Sudeste da Ásia, e que dispersou por países da América do Norte, Europa e América do Sul com potencial de se estabelecer na África e Oceania (DOS SANTOS et al. 2017). Essa espécie é considerada praga de frutos de epicarpo delgado como amora, cereja, framboesa, mirtilo, morango, entre outros. O status de praga se deve a presença de um ovipositor robusto, esclerotizado e serreado, que possibilita a oviposição em frutos sadios, em amadurecimento ou maduros. $O$ desenvolvimento das larvas no mesocarpo causa o apodrecimento dos frutos (SCHLESENER et al. 2017a).

O Brasil é um país de proporções continentais, que possui os mais variados tipos de climas e disponibilidade de substrato para criação de D. suzukii (SCHLESENER et al. 2014, 2015). No Brasil a espécie já foi registrada no Rio Grande do Sul, Santa Catarina (DEPRÁ et al. 2014), no Paraná (GEISLER et al. 2015), Minas Gerais (ANDREAZZA et al. 2016), Rio de Janeiro (BITNER-MATHÉ et al. 2014), Distrito Federal (PAULA et al. 2014) e São Paulo (VILELA \& MORI 2014). Medidas de controle já estão sendo Rev. Ciênc. Agrovet., Lages, SC, Brasil (ISSN 2238-1171) 526 
desenvolvidas no Brasil, com destaque para o controle biológico (GARCIA et al. 2017), técnica de inseto estéril (KRÜGER et al. 2018) e controle químico (SCHLESENER et al. 2017b).

Em Santa Catarina, a espécie foi registrada nos municípios de Botuverá, Nova Veneza e Lages (DEPRÁ et al. 2014, SOUZA et al. 2017). Dessa forma, é importante que estudos relatem a ocorrência da praga e a ampliação da distribuição geográfica de $D$. suzukii, o que permitirá estudos de modelagem de nicho da espécie e medidas regionais de manejo da praga. $O$ presente trabalho teve como objetivo registar a ampliação da ocorrência da espécie na região Serrana de Santa Catarina.

Dessa forma, foi realizada amostragem de $D$. suzukii em área de produção de morango (Fragaria $x$ ananassa) cultivar Albion em sistema de produção semi-hidropônico, no município de Frei Rogério, SC (27ํㅜㄴ $10^{\prime} 29^{\prime \prime} \mathrm{S}$ e $50^{\circ} 48^{\prime} 17^{\prime \prime} \mathrm{W}$ ) a $950 \mathrm{~m}$ de altitude (Figura 1).

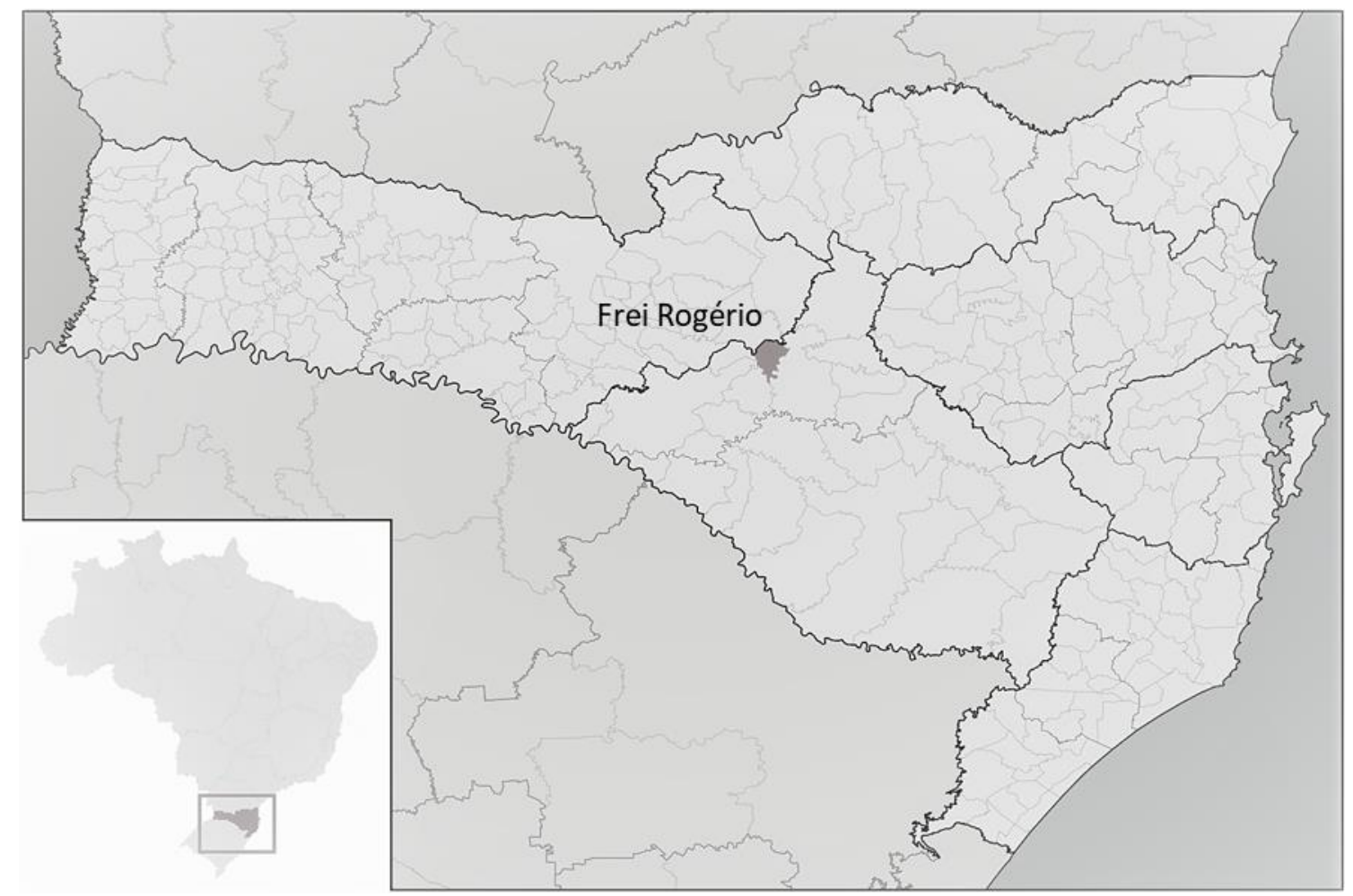

Figura 1. Local de coleta de Drosophila suzukii na região Serrana Catarinense. Em destaque à esquerda: 0 estado de Santa Catarina. (Imagem: Raphael Lorenzeto de Abreu).

Figure 1. Collect locality of Drosophila suzukii in the Catarinense Serrana region. Left highlight: The state of Santa Catarina. (Image: Raphael Lorenzeto de Abreu).

Para tal foram utilizadas armadilhas tipo caça-mosca confeccionadas em PET reciclado iscadas com vinagre de maçã e água na proporção $1: 2$, penduradas $50 \mathrm{~cm}$ acima das plantas de morango. As armadilhas $(n=21)$ monitoraram 8.600 plantas de morangueiro durante 10 dias (monitoradas no quinto e décimo dias) após serem colocadas nas estufas (dados climatológicos médios para o município no período: temperatura média $14,73^{\circ} \mathrm{C}$ e umidade relativa $87 \%$ EPAGRI/CIRAM). Cada armadilha teve seu conteúdo cuidadosamente removido, preservado em álcool $70 \%$ e posteriormente avaliado em laboratório com auxílio de um microscópio estereoscópio para verificar a presença da praga.

Foram encontrados machos $(n=04)$ e fêmeas $(n=98)$ de D. suzukii (Figura 2), possivelmente oriundos de práticas de manejo como raleio e plantas adjacentes, visto que não foram encontradas larvas da mosca nos frutos amostrados até o momento.

A identificação da espécie foi obtida comparando os espécimes coletados com a literatura disponível (SCHLESENER et al. 2015) e confirmada pelo Dr. Flávio Roberto Mello Garcia do Laboratório de Ecologia de Insetos na Universidade Federal de Pelotas (LABEI - UFPEL). Se considerarmos a amplitude térmica e altitude das localidades onde já foi relatada a presença da praga no estado de Santa Catarina (DEPRÁ et al. 2014, SOUZA et al. 2017), podemos observar que não existe um perfil geográfico preferencial para a praga. O que pode ser claramente explicado pela tolerância de $D$. suzukii a baixas temperaturas, o que é incomum nas demais espécies do gênero. O limite inferior de temperatura suportado pela praga é amplo, sendo que em cerca de $10{ }^{\circ} \mathrm{C}$ há redução de mobilidade e atividades reprodutivas, no entanto, as temperaturas letais 
podem ser ainda mais baixas dependendo da origem da população e estação de coleta (STEPHENS et al. 2015). Embora o mecanismo pelo qual D. suzukii permaneça em diapausa durante os períodos em que a temperatura está abaixo do seu limiar térmico inferior, o que se sabe até o momento é que estes indivíduos pausam suas atividades principalmente reprodutivas, até que as condições retornem ao ótimo para seu desenvolvimento (DALTON et al. 2011).
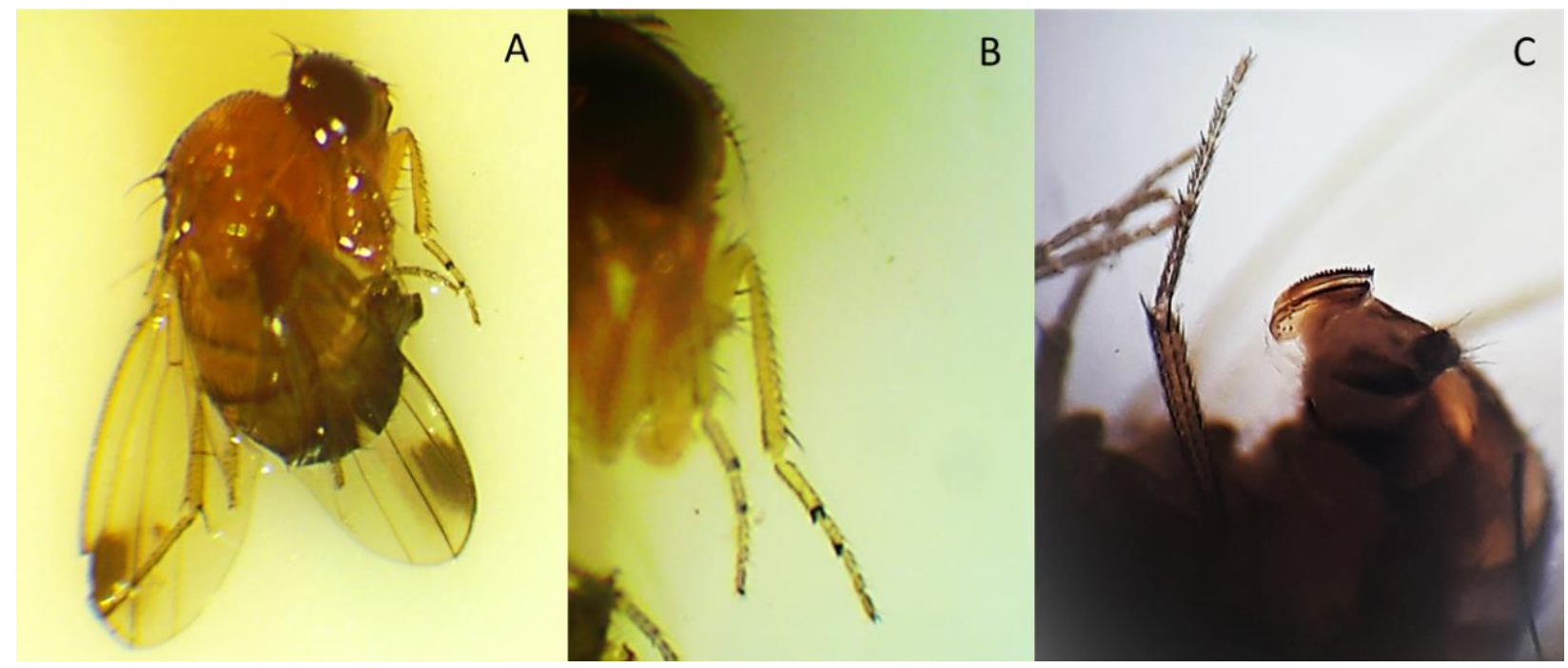

Figura 2. Drosophila suzukii coletadas na região Serrana de Santa Catarina. A) Macho, com detalhe das manchas escuras no ápice das asas; B) Detalhe da fileira de cerdas no primeiro e segundo segmento tarsal do primeiro par de pernas; C) Fêmea, detalhe do ovipositor duplamente serreado.

Figure 2. Drosophila suzukii collected in the Serrana Region of Santa Catarina. A) Male, spotted wings B) Male, detail: combs on the foretarsi C) Female, ovipositor with two rows of serrated teeth.

A ocorrência da praga em mais um ponto no estado de Santa Catarina, reafirma o potencial de dispersão de $D$. suzukii relatado para extensões da costa do Atlântico, sobretudo sul do Brasil e países cujo território brasileiro faz fronteira, como o Uruguai, Paraguai e Argentina. Os fatores abióticos encontrados nessas faixas de latitude variam em intervalos de temperatura média entre 5 e $20^{\circ} \mathrm{C}$ o que vai de encontro ao ótimo de desenvolvimento da praga, e essa condição está frequentemente associada ao regime anual de chuvas de até $2.500 \mathrm{~mm}$ determinante para a distribuição da espécie (DOS SANTOS et al. 2017), características climáticas que foram relatadas nesse trabalho, região da Serra Catarinense.

As condições de clima associadas ao potencial de distribuição da espécie, nos leva a crer numa maior dispersão da população da praga, expandindo-se para outras regiões do estado e da região sul do Brasil, em virtude de atender as condições da praga, mas também de possuir as condições edafoclimáticas para o cultivo de seus hospedeiros. Além disso, vale destacar que a disseminação de $D$. suzukii por meio de características biológicas intrínsecas da população, mas de dispersão passiva, como o transporte de frutos com ovos e larvas pelo homem, por exemplo, o que intensificaria esse processo.

A ocorrência de $D$. suzukii no município de Frei Rogério, SC é um fato relevante para a construção dos modelos de predição do aumento e avanço das populações brasileiras da drosófila-da-asa-machada e com isso buscar a compreensão do panorama ecológico e comportamental de dispersão e evolução da praga, estando ela aumentada pela disponibilidade hospedeira associada às condições abióticas ou ainda com a influência de atividades antrópicas devido ao alto potencial de dano da praga.

\section{AGRADECIMENTO}

Somos gratos pela confiança e disponibilidade dos proprietários da Oishi's Fruits. Ao CNPq pela Bolsa de Produtividade em Pesquisa de FRMG.

\section{REFERÊNCIAS}

ANDREAZZA F et al. 2016. Drosophila suzukii (Diptera: Drosophilidae) arrives at Minas Gerais State, a Main Strawberry Production Region in Brazil. The Florida Entomologist 99: 796-798.

BITNER-MATHÉ BC et al. 2014. Drosophila suzukii has been found in tropical Atlantic rainforest in southeastern Brazil. Drosophila Information Service 97: 136-137.

DALTON TD el al. 2011. Laboratory survival of Drosophila suzukii under simulated winter conditions of the Pacific 
Northwest and seasonal field trapping in five primary regions of small and stone fruit production in the United States. Pest Management Science 67: 1368-1374.

DEPRÁ M et al. 2014. The first records of the invasive pest Drosophila suzukii in South American Continent. Journal of Pest Science 87: 379-383.

DOS SANTOS LA et al. 2017. Global potential distribution of Drosophila suzukii (Diptera, Drosophilidae). Plos One 12: 0174318.

GARCIA FRM et al. 2017. Biological Control of Drosophila suzukii (Diptera, Drosophilidae): State of the Art and Prospects. In: Lewis Davenport. (Org.). Biological Control: Methods, Applications and Challenges. Hauppauge: Nova Science Publishers. p.1-27.

GEISLER FCS et al. 2015. Primeiro registro de Drosophila suzukii (Matsumura, 1931) (Diptera: Drosphilidae) para o Estado do Paraná, Brasil e de novos hospedeiros. Revista de Ciências Ambientais 9: 125-129.

KRÜGER AP et al. 2018. Effects of Irradiation Dose on Sterility Induction and Quality Parameters of Drosophila suzukii (Diptera: Drosophilidae). Journal of Economic Entomology 111: 741-746

PAULA MA et al. 2014. First record of Drosophila suzukii in the Brazilian Savanna. Drosophila Information Service 97:113-115.

SCHLESENER DCH. et al. 2014. Mosca-da-cereja (Drosophila suzukii): Uma nova ameaça para a fruticultura brasileira. Cultivar 12: 6-8.

SCHLESENER DCH. et al. 2015. Drosophila suzukii: nova praga para a fruticultura brasileira. O Biológico 77: 47-54.

SCHLESENER DCH et al 2017a. Drosophila suzukii (Matsumura, 1931) (Diptera, Drosophilidae): Biology, Ecology and Control. 1.ed. Pelotas: UFPel.

SCHLESENER DCH et al. 2017b. Effects of insecticides on adults and eggs of Drosophila suzukii (Diptera, Drosophilidae). Revista Colombiana de Entomología 43: 208-214.

SOUZA GK et al. 2017 Acca sellowiana (Myrtaceae): A New Alternative Host for Drosophila suzukii (Diptera: Drosophilidae) in Brazil. Florida Entomologist 100: 190-191.

STEPHENS AR et al. 2015 Cold Hardiness of Winter-Acclimated Drosophila suzukii (Diptera: Drosophilidae) Adults. Environmental Entomology 44: 1619-1626.

VILELA C \& MORI L. 2014. The invasive spotted-wing Drosophila (Diptera, Drosophilidae) has been found in the city of São Paulo (Brazil). Revista Brasileira de Entomologia 58: 371-375. 